Although we were unable to culture $S$. marcescens from any products, based on the strength of the epidemiological data and molecular results, we concluded that the outbreak of S. marcescens bacteremia was most likely associated with contaminated prefilled heparin flushes. Prefilled heparin syringes were a common product shared by all cases at our institution, and this was also the only product shared by other institutions. Additionally, none of the cases were in the neonates or adult patient populations, which have limited use of prefilled heparin flushes at the concentrations used in pediatric patients. Although S. marcescens was not found in any cultures from any prefilled heparin flushes, Bacillus spp were identified from several different lots, suggesting a problem with sterility during manufacturing. ${ }^{2,8}$

In conclusion, our experience highlights key features of a successful epidemiologic outbreak investigation including rapid identification and reporting to public health of a suspected outbreak, investigation of all inpatient and outpatient clusters of bacteremia by infection preventionists, the essential role of molecular typing, and timely communication via a pediatric national network listserv. Furthermore, this investigation underscores the importance of pursuing product-associated outbreaks supported by strong epidemiologic data despite the lack of culture-proven product contamination.

Author ORCIDs. Kelly West (iD) https://orcid.org/0000-0001-7938-9239

Acknowledgments. None.

Financial support. No financial support was provided relevant to this article.
Conflicts of interest. All authors report no conflicts of interest relevant to this article.

\section{References}

1. Archibald LK, Corl A, Shah B, et al. Serratia marcescens outbreak associated with extrinsic contamination of $1 \%$ chlorxylenol soap. Infect Control Hosp Epidemiol 1997;18:704-709.

2. Blossom D, Noble-Wang J, Su J, et al. Multistate outbreak of Serratia marcescens bloodstream infections caused by contamination of prefilled heparin and isotonic sodium chloride solution syringes. Arch Intern Med 2009;169:1705-1711.

3. Sunenshine RH, Tan ET, Terashita DM, et al. A multistate outbreak of Serratia marcescens bloodstream infection associated with contaminated intravenous magnesium sulfate from a compounding pharmacy. Clin Infect Dis 2007;45:527-533.

4. Iosifidis E, Farmaki E, Nedelkopoulou N, et al. Outbreak of bloodstream infections because of Serratia marcescens in a pediatric department. Am J Infect Control 2012;40:11-15.

5. Manning ML, Archibald LK, Bell LM, Banerjee SN, Jarvis WR. Serratia marcescens transmission in a pediatric intensive care unit: a multifactorial occurrence. Am J Infect Control 2001;29:115-119.

6. Ostrowsky BE, Whitener C, Bredenberg HK, et al. Serratia marcescens bacteremia traced to an infused narcotic. $N$ Engl J Med 2002;346: 1529-1537.

7. Jones BL, Gorman LJ, Simpson J, et al. An outbreak of Serratia marcescens in two neonatal intensive care units. J Hosp Infect 2000;46:314-319.

8. Su JR, Blossom DB, Chung W, et al. Epidemiologic investigation of a 2007 outbreak of Serratia marcescens bloodstream infection in Texas caused by contamination of syringes prefilled with heparin and saline. Infect Control Hosp Epidemiol 2009;30:593-595.

\title{
A phylogenetic study of Elizabethkingia anophelis bloodstream isolates obtained from inpatients at a single medical center
}

\author{
Rajesh Kumar Sahoo $\mathrm{PhD}^{1}$, Saubhagini Sahoo MSc${ }^{1}$, Aradhana Das ${ }^{1}$, Mahendra Gaur MTech ${ }^{1}$, \\ Debadatta Bhanjadeo $\mathrm{MD}^{2}$, Prasanjeeta Panda $\mathrm{MSc}^{1}$ and Enketeswara Subudhi $\mathrm{PhD}^{1}$ \\ ${ }^{1}$ Centre for Biotechnology, Siksha 'O' Anusandhan University, Bhubaneswar, Odisha, India and 'Department of Health and Family Welfare, Government of \\ Odisha, Bhubaneswar, Odisha, India
}

To the Editor-Elizabethkingia anophelis is a rapidly emerging nosocomial pathogen reported to cause bacteremia in immunecompromised elderly people and neonates., ${ }^{1,2}$ The unknown pathogenesis and unclear resistance mechanism of E. anophelis and their phenotypic similarity to E. meningoseptica mislead and complicate the infection management of this pathogen, resulting in treatment failure. Inherent resistance to multiple classes of drugs and absence of an antibiotic sensitivity profile standard for this bacterium makes empirical treatment nearly impossible. Elizabethkingia anophelis bacteremia has recently been

Author for correspondence: Dr Enketeswara Subudhi MTech, $\mathrm{PhD}$, Centre for Biotechnology, School of Pharmaceutical Sciences, Siksha 'O' Anusandhan University, Kalinga Nagar, Ghatikia, Bhubaneswar, Odisha-751003 (IN). E-mail: enketeswarasubudhi@ soa.ac.in

Cite this article: Sahoo RK, et al. (2019). A phylogenetic study of Elizabethkingia anophelis bloodstream isolates obtained from inpatients at a single medical center. Infection Control \& Hospital Epidemiology, 40: 1202-1204, https://doi.org/10.1017/ ice. 2019.213 considered clinically significant, leading to high morbidity and mortality that has been mistakenly attributed to E. meningoseptica because of their phenotypic similarity. ${ }^{2}$ Molecular epidemiological analyses of recent Elizabethkingia bacteremia infections and outbreaks have been conducted in United States, Singapore, China, and Korea. These outbreaks were predominated by E. anopheles. ${ }^{3-6}$ This finding warrants the implementation of molecular typing for an accurate diagnosis to guide appropriate antibiotic regimen instead of relying solely on conventional phenotypic identification with a compact automated VITEK-2 system, which uses a factory default database and lacks timely amendments. ${ }^{3,5}$

In the first report of an outbreak in a tertiary healthcare center of Eastern India, the clinical and molecular epidemiology of 9 bacteremia episodes during 2 months of surveillance from August to September 2017 were identified as E. meningoseptica by the VITEK-2 system. These findings were genetically validated by species-specific markers, such as lipid-A disaccharide synthase 


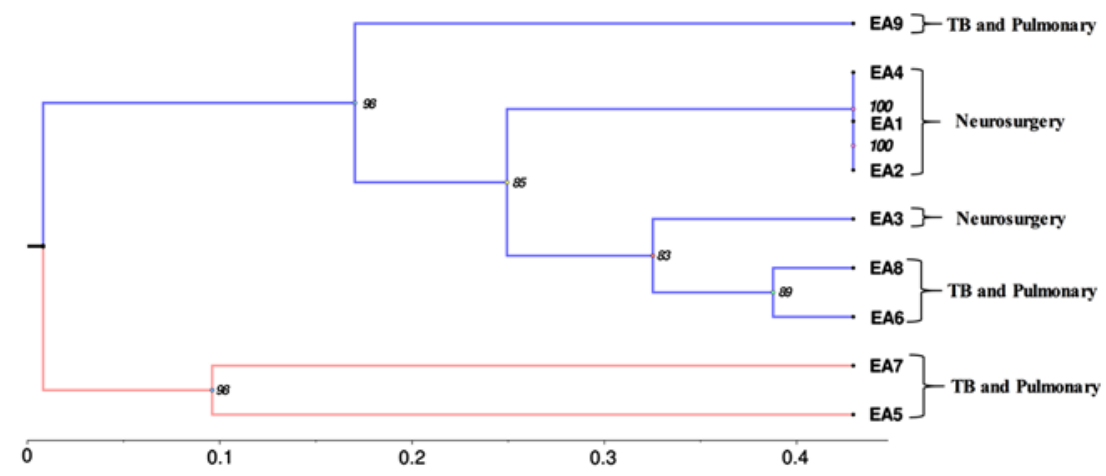

Fig. 1. The phylogenetic tree was developed using REP and (GTG)5 PCR. We observed close clustering among 9 isolates as they relate to the same in-patient department (as labeled): EA1 and EA2 with EA4; EA6 with EA8; and EA5 with EA7. A phylogenetic tree was constructed based on Jaccard similarity coefficient. Hierarchical clustering was performed by hclust function ( $R$ Core Team stats 3.5.3) using the unweighted pair group method with arithmetic mean (UPGMA) method, and the tree was cut into an optimal number of clusters along with bootstrapping 1,000 times (Pvclust version 2.0).

gene for E. anophelis and sodium-proton antiporter for E. meningoseptica, ${ }^{5}$ and $16 \mathrm{~s}$ rRNA gene sequencing. An antibiotic susceptibility study was conducted using the VITEK-2 compact automated system (BioMerieux) with the GN-AST-N280 card. Sensitivity was interpreted according to Clinical Laboratory Standards Institute (CLSI) guidelines (2013). ${ }^{7}$ The clonal relatedness among 9 isolates was investigated using repetitive-element polymerase chain reaction (rep-PCR) and (GTG) 5 PCR according to the method described by Adiguzel et al. ${ }^{8}$

Nonrepeated Elizabethkingia spp (EA1-9) were isolated from 9 inpatients, and we analyzed the demographic data, clinical characteristics, and outcomes for these cases (Table S1 online). In these 9 cases, Elizabethkingia bacteremia prevailed mostly among elderly people $(\mathrm{n}=8$; median age 52 years), but 1 patient was a 2 -year-old child. The male: female ratio among these patients was 7:2 (Table S1 online). Overall, 5 Elizabethkingia isolates were obtained from blood; the rest were obtained from tracheal aspiration $(\mathrm{n}=3)$ and cerebrospinal fluid $(n=1)$. All of these patients were reported as having hospitalacquired, clinically significant bacteremia, with a high mortality rate (33.3\%). Of these 9 patients, 3 died within 1 month of their hospital stay despite treatment with antibiotics (eg, quinolones, penicillin, cephalosporins, carbapenems, etc, either alone or in combination) due to several associated complications: pneumonia, lower respiratory infection, meningitis, acute kidney injury, and metabolic encephalopathy, etc.

These isolates showed resistance to different groups of antibiotics with varying percentages ranging from $\sim 80 \%$ to $100 \%$ (Table S2 online). However, the highest susceptibility was found against tigecycline and piperacillin-tazobactam, which corroborates the previous reports ${ }^{3,4,9}$ except isolate EA1, which matched a single study from China. ${ }^{6}$ The resistance profile against levofloxacin was analogous to isolates identified in Korea and Wisconsin. ${ }^{3,4}$ However, the alteration of the antibiotic resistance profile depends generally on different types of stress on different sources of Elizabethkingia isolates. ${ }^{6}$

All 9 isolates were identified as E. meningoseptica by the VITEK-2 compact automated system. Because the identification of Elizabethkingia spp has been reported to be misleading using the VITEK 2 and MALDI-TOF MS systems, ${ }^{2}$ these samples were subjected to genotypic validation. However, upgrading the VITEK-2 system with better antibiotic sensitivity profiles, updating the CLSI guidelines, and expanding the database for MALDI-TOF mass spectra of $E$. anophelis will improve their proper identification. All 9 Elizabethkingia spp showed amplification of lipid-A disaccharide synthase gene, a species-specific primer of E. anopheles, and were further confirmed to be E. anophelis by $16 \mathrm{~s}$ rRNA gene sequence analysis (GenBank accession no: MH121154MH121158, MN038050-MN0380053). Rep PCR- and (GTG)5 PCR-based phylogenetic analysis of 9 isolates revealed a close clustering of EA1 and EA2 with EA4; EA6 with EA8; and EA5 with EA7. These findings explain the considerable clonal similarity among E. anophelis isolates belonging to the same in-patient departments (Fig. 1).

This study is the first molecular epidemiological report on a bacteremia outbreak of India with prevalence of E. anophelis bacteria establishing E. meningoseptica to be the more remote cause of bacteremia infection. However, future prospective studies with population-based data over longer surveillance periods should be performed to determine the prevalence and incidence of $E$. anophelis bacteremia. A repeated molecular epidemiological study should be employed for accurate diagnosis and appropriate treatment regimen.

Acknowledgment. The authors thank Dr N. K. Debata, Department of Microbiology, IMS and SUM Hospital, Siksha 'O' Anusandhan University, Bhubaneswar, Odisha, India for providing bacterial samples and related information. The authors are also grateful to Prof (Dr) S. C. Si, Dean, Centre for Biotechnology and Prof (Dr) M. R. Nayak, President, Siksha 'O' Anusandhan University, for providing infrastructure and encouragement throughout the study.

Financial support. This study was supported by the Science and Engineering Research Board (grant no. EMR/2016/006732), New Delhi, India.

Conflicts of interest. The authors declare that they have no conflict of interest.

Supplementary material. To view supplementary material for this article, please visit https://doi.org/10.1017/ice.2019.213

\section{References}

1. Lau SKP, Wu AKL, Teng JLL, et al. Evidence for Elizabethkingia anophelis transmission from mother to infant, Hong Kong. Emerg Infect Dis 2015;21:232-241.

2. Lau SKP, Chow WN, Foo CH, et al. Elizabethkingia anophelis bacteremia is associated with clinically significant infections and high mortality. Sci Rep 2016;6:8-17.

3. Han MS, Kim H, Lee Y, et al. Relative prevalence and antimicrobial susceptibility of clinical isolates of Elizabethkingia species based on 16S rRNA gene sequencing. J Clin Microbiol 2017;55:274-280.

4. Perrin A, Larsonneur E, Nicholson AC, et al. Evolutionary dynamics and genomic features of the Elizabethkingia anophelis 2015 to 2016 Wisconsin outbreak strain. Nat Comm 2017;8. 
5. Chew KL, Cheng B, Lin RTP, Teo JWP. Elizabethkingia anophelis is the dominant Elizabethkingia species found in blood cultures in Singapore. J Clin Microbiol 2018;56:1-3.

6. Wang M, Gao H, Lin N, et al. The antibiotic resistance and pathogenicity of a multidrug-resistant Elizabethkingia anophelis isolate. MicrobiologyOpen 2019;e804.

7. Clinical and Laboratory Standards Institute. Performance Standards for Antimicrobial Susceptibility Testing, 29th edition. Wayne, PA: CLSI; 2013.
8. Adiguzel A, Ozkan H, Baris O, Inan K, Gulluce M, Sahin F. Identification and characterization of thermophilic bacteria isolated from hot springs in Turkey. J Microbiol Meth 2009;79:321-328.

9. Lin JN, Lai CH, Yang CH, Huang YH, Lin HH. Genomic features, phylogenetic relationships, and comparative genomics of Elizabethkingia anophelis strain EM361-97 isolated in Taiwan. Sci Rep 2017;7:8.

\title{
Impact of neutropenia on central venous catheter-related bloodstream infections in patients with hematological malignancies at the time of central venous catheter insertion: A matched-pair analysis
}

\author{
Daniela Tölle $M D^{1}$, Marcus Hentrich $\mathrm{MD}^{2}$, Benedikt W. Pelzer $\mathrm{MD}^{3}$, Pierre Kremer $\mathrm{MD}^{4}$, Sabine Einhell $\mathrm{MD}^{5}$, \\ Sebastian Schulz MD², Boris Böll MD², Jens Panse MD ${ }^{5}$, Martin Schmidt-Hieber MD ${ }^{4}$, Daniel Teschner MD ${ }^{1}$ \\ and Enrico Schalk $M D^{6}$ (i) \\ ${ }^{1}$ Department of Hematology, Medical Oncology, and Pneumology, University Medical Center of the Johannes Gutenberg University Mainz, Mainz, Germany, \\ ${ }^{2}$ Department of Hematology and Oncology, Red Cross Hospital Munich, Munich, Germany, ${ }^{3}$ Department of Internal Medicine I, Center for Integrated Oncology \\ Aachen Bonn Cologne Dusseldorf, University Hospital of Cologne, Cologne, Germany, ${ }^{4}$ Clinic of Hematology and Oncology, Carl-Thiem Hospital Cottbus, Cottbus, \\ Germany, ${ }^{5}$ Department of Oncology, Hematology, Hemostaseology and Stem Cell Transplantation, Medical Faculty, University Hospital RWTH Aachen, Aachen, \\ Germany and ${ }^{6}$ Department of Hematology and Oncology, Medical Center, Otto-von-Guericke University Magdeburg, Magdeburg, Germany
}

To the Editor-Central venous catheter (CVC)-related bloodstream infections (CRBSIs) are potentially preventable complications associated with high morbidity, especially in patients with hematological malignancies. ${ }^{1}$ Neutropenia is one of the most important risk factors for CRBSI ${ }^{1,2}$ because of insufficient immune control of the patient's microbial flora or pathogens when absolute neutrophil counts (ANC) are $<500 / \mu \mathrm{L}^{3,4}$ Neutropenia as a risk factor for CRBSI is only well defined for the time of CRBSI onset. ${ }^{5,6}$ So far, no reliable data are available that address the impact of neutropenia on CRBSI at the time of CVC insertion. This impact is of special interest for hematology patients because CVCs are often inserted during neutropenia either due to the underlying malignancy, like acute myeloid leukemia (AML), or after application of chemotherapy. In addition, CVC reinsertions after CVC removal due to CRBSI are also common, especially during longlasting neutropenia, for example, after remission-inducing chemotherapy in AML patients or after conditioning therapies before hematopoietic stem-cell transplantation (HSCT). Therefore, we aimed to investigate the impact of neutropenia on subsequent CRBSI at the time of insertion of short-term, nontunneled CVCs in adult patients with hematological malignancies.

We analyzed data from the prospective multicenter SECRECY study (German Clinical Trial Register, no. DRKS00006551), a CRBSI registry conducted in 6 German hematology and oncology centers, including the aforementioned group of high-risk patients

Author for correspondence: Enrico Schalk, MD, Otto-von-Guericke University Magdeburg Medical Center, Department of Hematology and Oncology, Leipziger Str 44, D-39120 Magdeburg, Germany. E-mail: enrico.schalk@med.ovgu.de

Cite this article: Tölle D, et al. (2019). Impact of neutropenia on central venous catheter-related bloodstream infections in patients with hematological malignancies at the time of central venous catheter insertion: A matched-pair analysis. Infection Control \& Hospital Epidemiology, 40: 1204-1206, https://doi.org/10.1017/ice.2019.224 receiving AML induction or HSCT. Inclusion criteria encompassed short-term, nontunneled jugular and subclavian vein CVCs with $\geq 1$ day in situ, and CRBSI was classified according to the 2012 Infectious Diseases Working Party (AGIHO) of the German Society for Hematology and Medical Oncology (DGHO) CRBSI definition. ${ }^{2}$ Only CRBSIs classified as definite or probable were considered. Hence, we identified 2,368 patients with a total follow-up of 37,932 CVC days. In 2,158 cases $(91.1 \%)$, the underlying diseases were hematological malignancies. Among them, we identified 406 cases $(17.1 \%)$ with neutropenia defined as ANC $<500 / \mu \mathrm{L}$ or white blood cell counts $<1,000 / \mu \mathrm{L}$ at the time of CVC insertion. Of 406 cases, 400 could be matched for age, sex, underlying disease, site of CVC insertion, use of chlorhexidine-coated CVC dressings, use of chlorhexidine-, antimicrobial- or silver sulfadiazine-coated CVCs, and complications during CVC insertion.

The median age of the patients was 59 years, and $60.3 \%$ were male (Table 1). Approximately $75 \%$ of CVCs were inserted in patients suffering from acute leukemia, and almost all CVCs were placed in the jugular vein (388 of 400, 97.0\%). In a minority of patients, coated CVCs were inserted (23 of $400,5.8 \%$ ), whereas in half of the cases chlorhexidine-coated CVC dressings were used (196 of 400, 49.0\%). Known high-risk CVC features (ie, male sex, complicated CVC insertion, diagnosis of AML, multiple myeloma, or non-Hodgkin lymphoma ${ }^{7}$ ) were present in half of the patients (203 of 400, 50.8\%). The median CVC time in situ was nonsignificantly shorter in CVCs inserted during neutropenia compared to CVCs in nonneutropenic patients (14 vs 18 days; $P=.39$ ). We found no differences in CRBSI rates comparing neutropenic to nonneutropenic patients (45 of 400 [11.3\%] vs 50 of 400 [12.5\%]; $P=.66)$. However, median time to CRBSI diagnosis was shorter in patients who received the CVC in neutropenia 